\title{
The combination of system dynamics and game theory in analyzing oligopoly markets
}

\author{
Ali Mohammadi ${ }^{\mathrm{a}}$, Alinaghi Mosleh Shirazi ${ }^{\mathrm{b}}$, Ahmad Talebnezhad ${ }^{\mathrm{c}}$, Ahmad Sadraee Javaheri ${ }^{\mathrm{b}}$ and \\ Ehsan Javanmardi ${ }^{*}$
}

\begin{abstract}
${ }^{a}$ Professor, School of Economics, Management and Social Sciences, University of Shiraz, Shiraz, Iran
${ }^{b}$ Associate Professor, School of Economics, Management and Social Sciences, University of Shiraz, Shiraz, Iran

${ }^{c}$ Assistant Professor, School of Economics, Management and Social Sciences, University of Shiraz, Shiraz, Iran

${ }^{d}$ Ph.D. Student, School of Economics, Management and Social Sciences, University of Shiraz, Shiraz, Iran

\section{CH R O N I C L E}

A B S T R A C T
\end{abstract}

Article history:

Received October 28, 2015

Received in revised format

November 28, 2015

Accepted January 28, 2016

Available online

February 2, 2016

Keywords:

Systems dynamic

Game theory

Oligopoly market

\begin{abstract}
In this paper, we present a hybrid method of game theory and dynamic systems to study the behavior of firms in an oligopoly market. The aim of this study is to build a model for an oligopoly game on the basis of feedback loops and system dynamics approach and to solve the resulted problems under some special conditions where traditional game theory methods are unable to handle. The method includes a combination of qualitative methods including interviews with industry experts to prepare the model and quantitative methods of system dynamics, simulation methodologies and game theory. The results indicate that competitive behavior and the important parameters such as volume of demand, interest rates and price fluctuation will be stabilized after a transition period.
\end{abstract}

\section{Introduction}

When an entity's profit does not necessarily depend on his/her behavior, but it could be influenced by the behavior of one or more other entities, and the decisions of others, both could have positive and negative impacts on his/her profits, a game between two or more entities are formed (Ahmed \& Hegazi, 2006). In managing games, different strategies could be considered. Feedback Stackelberg strategies, for instance, are considered for two-person linear multi-stage games with quadratic performance criteria and noisy measurements (Castanon \& Athans, 1976). In the world of commerce and business, there is always an ongoing game and whenever there is only limited number of suppliers for a particular product, an oligopoly game is formed. In this structure, the activity of each seller will affect the behavior of other vendors. Another point in an oligopoly market structure is that there is an interdependency between the firms compared with the other markets and this is a natural consequence of the limited number of suppliers. The primary objective of this study is to evaluate the competitive behavior of the firms in an oligopolistic market based on a hybrid of system dynamics and game theory

* Corresponding author

E-mail address: javanmardi.ehsan@gmail.com (E. Javanmardi) 
(Akiyama \& Kaneko, 2002). Baiardi et al. (2015) proposed an evolutionary model of oligopoly competition where agents could choose between various behavioral rules to make decisions on productions. Merlone and Szidarovszky (2015) investigated dynamic oligopolies with contingent workforce and investment costs. Zhang et al. (2015) presented a game-theoretic economic operation of residential distribution system with high participation of distributed electricity prosumers. They determined a new roles of utilities and distributed electricity prosumers in the future retail electricity market. The game-theoretic algorithms were implemented to detect the retail electricity market price by considering the group coalition scenarios of multiple electricity prosumers. Lambertini and Mantovani (2014) proposed a feedback equilibria in a dynamic renewable resource oligopoly. They examined a recent literature on productive asset exploitation describing a differential oligopoly game of resource extraction under static, linear feedback and nonlinear feedback strategies, where they permitted for the possibility of resource exhaustion. They reported that, first, feedback rules could entail resource exhaustion for a finite number of firms. In addition, feedback strategies were more aggressive than static ones as long as the resource stock was big enough, in accordance with the acquired view based on the traditional pre-emption argument associated with feedback information. Akiyama and Kaneko (2000) presented a theoretical framework called dynamical systems game, in which the game itself could be changed due to the effect of players' behaviors and states. Asker (2007) formulated a dynamical multi-team Cournot game for a renewable resource.

\section{The proposed study}

The main problem with the majority of current models is to predict the future, in fact, in most games the environment is assumed to be constant. However, in real-world, most players change their strategies based on different events. In fact, each player must consider different circumstances and make his/her decision according to the consequences, which could happen in future. A dynamic game can be defined as follows (Nash, 1950, 1951; Mingers, 2004),

$$
G:(E(t), S(t)) \rightarrow(E(t+1), S(t+1))
$$

where $G$ represents a game, $E$ denotes the status of environment and $S$ is associated with status of each player. Finally, $t$ indicates the status of the game over time. Therefore, we have

$$
\begin{aligned}
& u: \quad(E(t), S(t)) \rightarrow\left(E(t)^{\prime}, S(t)^{\prime}\right) \\
& v: \quad\left(E(t)^{\prime}, S(t)^{\prime}, O(t)\right) \rightarrow(E(t+1), S(t+1)) \\
& G: \quad u \circ v
\end{aligned}
$$

Here $u$ represents natural laws, $v$ denotes the effects of players' actions and $o$ is associated with actions that players accomplish. Each player setup his action as follows,

$$
Z^{i}:(E(t), S(t)) \rightarrow\left(O^{i}(t)\right)
$$

Here $i$ represents the player, $Z$ demonstrates the mechanism of decision making and $O$ represents action plans with $Z=\left\{Z^{1}, Z^{2}, \ldots, Z^{n}\right\}$. Thus,

$$
Z:(E(t), S(t)) \rightarrow(O(t))
$$

Let $N=\{1,2, \ldots, n\}$ be the set of the number of players, $E$ represents the status of player, $S=\left\{s^{1}, s^{2}, \ldots, s^{n}\right\}$ and $O=\left\{o^{1}, o^{2}, \ldots, o^{n}\right\}$ represent the state and action of each player, respectively.

Fig. 1 demonstrates the structure of game in system dynamics. 


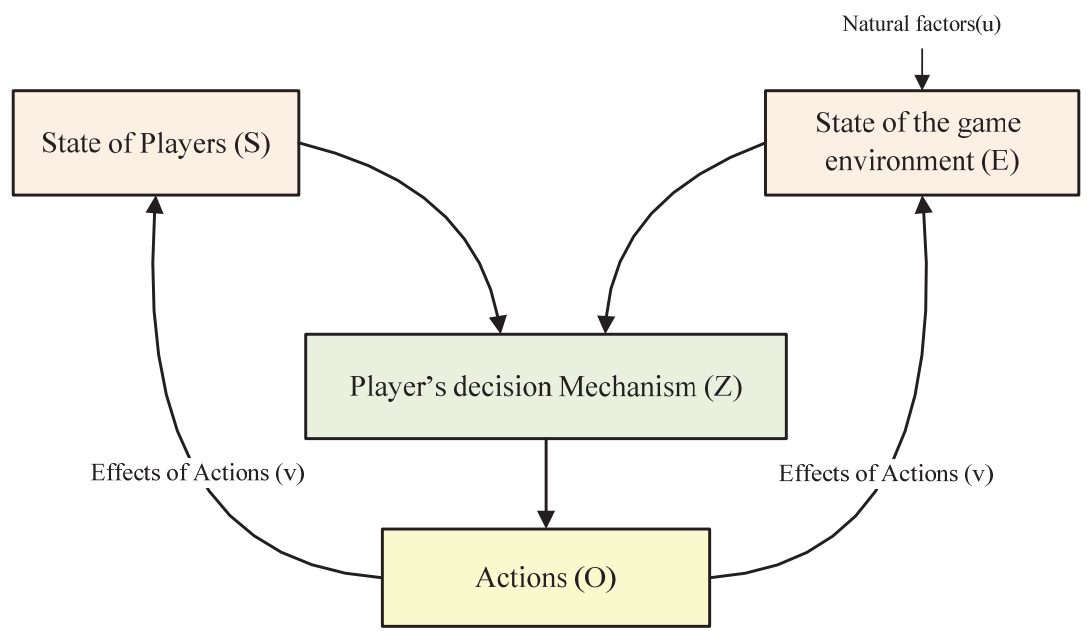

Fig. 1. The structure of system dynamics game

Here, each player tries to maximize his/her profit as follows,

$$
\pi=D \cdot P-C
$$

Here $P, D$ and $C$ are price, demand and cost, respectively. However, each player's behavior changes over the time so we have the following,

$$
E^{t}=\left\{D_{0}^{t}\right\}
$$

Let

$$
S_{i}^{t}=\left\{R_{i}^{t}, \frac{\pi_{i}^{t}}{\pi_{i}^{e}}\right\} \quad, \quad i=\{1,2, \ldots, n\}
$$

where $S_{i}^{t}$ and $R_{i}^{t}$ represent the status and market share of player $i$ at time $t, \pi_{i}^{t}$ and $\pi_{i}^{e}$ represent total and expected profit of player $i$ at time $t$, respectively. In general, we may expect to have a reverse relationship between demand and price as follows,

$$
\frac{d D_{i}}{d p_{i}} \leq 0 \quad, \quad \frac{d D_{i}}{d p_{-i}} \geq 0
$$

with

$$
\begin{aligned}
& \sum_{j=1}^{n} \frac{d D_{i}}{d P_{j}}<0 \\
& \sum_{j=1}^{n} \frac{d D_{j}}{d P_{i}}<0
\end{aligned}
$$

Therefore, the objective function of the proposed study can be summarized as follows,

$$
\begin{aligned}
& D=f\left(P, D_{0}\right) \\
& C=f(D, \alpha, \beta) \\
& \pi=D \cdot P-C=\left[D_{(P)} \cdot P\right]-\left[C_{(D, \alpha, \beta)}\right]
\end{aligned}
$$

Based on the Eqs. (5-13) we may show the relationships between different players in Fig. 2 as follows (West \& Lebiere, 2001), 


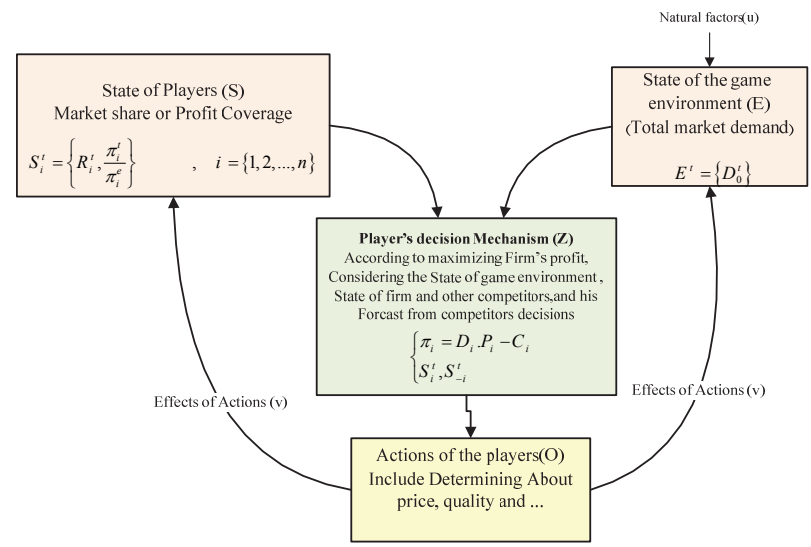

Fig. 2. The relationships between different players

\subsection{Modeling an oligopoly method}

As we have already stated, the revenue of each firm in an oligopoly market consists of the present profit plus the accumulated profitability over time, which can be calculated as follows,

$$
\begin{aligned}
& \operatorname{Max} \pi_{i}=D_{i} \cdot P_{i}-C_{i} \\
& B_{i}\left(O_{j}^{*}\right)=O_{i}^{*} \Rightarrow \frac{d \pi_{i}}{d O_{i}}=\nabla \pi_{i}=0
\end{aligned}
$$

where $O_{i}^{*}$ and $B_{i}\left(O_{j}^{*}\right)$ represent the optimum action and decision of player $i$ against player $j$, respectively. In linear form of demand, the relationship can be summarized as follows,

$$
\begin{aligned}
& D_{i}=D_{0 i}-\gamma_{i} \cdot P_{i}+\sum_{j \neq i}^{n} \gamma_{i j} \cdot P_{j} \quad, \quad \gamma_{i}, \gamma_{i j}, D_{0 i}>0 \\
& C_{i}=\beta_{i}+\alpha_{i} \cdot D_{i} \quad, \quad \beta_{i}, \alpha_{i}>0
\end{aligned}
$$

where $\alpha_{i}$ and $\beta_{i}$ represent variable and fixed costs of each player, respectively. Therefore, the Nash $(1950,1951)$ equilibrium equation is as follows,

$$
\pi_{i}=D_{i} \cdot P_{i}-C_{i} \Rightarrow B_{i}\left(P_{-i}^{*}\right)=P_{i}^{*} \quad \Rightarrow \frac{d \pi_{i}}{d P_{i}}=D_{0 i}-2 \gamma_{i} \cdot P_{i}+\sum_{j \neq i}^{n} \gamma_{i j} \cdot P_{j}+\alpha_{i} \cdot \gamma_{i}=0
$$

Solving Eq. (18) yields,

$$
\begin{gathered}
B_{i}\left(P_{-i}^{*}\right)=\frac{D_{0 i}+\sum_{j \neq i}^{n} \gamma_{i j} \cdot P_{j}^{*}+\alpha_{i} \cdot \gamma_{i}}{2 \gamma_{i}}=P_{i}^{*} . \text { In case we have only two competitors, we have, } \\
\left\{\begin{array} { l } 
{ B _ { 1 } ( P _ { 2 } ^ { * } ) = \frac { D _ { 0 1 } + \gamma _ { 1 2 } \cdot P _ { 2 } ^ { * } + \alpha _ { 1 } \cdot \gamma _ { 1 } } { 2 \gamma _ { 1 } } = P _ { 1 } ^ { * } } \\
{ B _ { 2 } ( P _ { 1 } ^ { * } ) = \frac { D _ { 0 2 } + \gamma _ { 2 1 } \cdot P _ { 1 } ^ { * } + \alpha _ { 2 } \cdot \gamma _ { 2 } } { 2 \gamma _ { 2 } } = P _ { 2 } ^ { * } }
\end{array} \Rightarrow \left\{\begin{array}{l}
P_{1}^{*}=\frac{2 \gamma_{2} \cdot D_{01}+2 \gamma_{2} \cdot \alpha_{1} \cdot \gamma_{1}+\gamma_{12} \cdot D_{02}+\gamma_{12} \cdot \alpha_{2} \cdot \gamma_{2}}{4 \gamma_{1} \gamma_{2}-\gamma_{12} \gamma_{21}} \\
P_{2}^{*}=\frac{2 \gamma_{1} \cdot D_{02}+2 \gamma_{1} \cdot \alpha_{2} \cdot \gamma_{2}+\gamma_{21} \cdot D_{01}+\gamma_{21} \cdot \alpha_{1} \cdot \gamma_{1}}{4 \gamma_{1} \gamma_{2}-\gamma_{12} \gamma_{21}}
\end{array}\right.\right. \\
\Rightarrow N\left(P_{1}^{*}, P_{2}^{*}\right)=\left(\frac{2 \gamma_{2} \cdot D_{01}+2 \gamma_{2} \cdot \alpha_{1} \cdot \gamma_{1}+\gamma_{12} \cdot D_{02}+\gamma_{12} \cdot \alpha_{2} \cdot \gamma_{2}}{4 \gamma_{1} \gamma_{2}-\gamma_{12} \gamma_{21}}, \frac{2 \gamma_{1} \cdot D_{02}+2 \gamma_{1} \cdot \alpha_{2} \cdot \gamma_{2}+\gamma_{21} \cdot D_{01}+\gamma_{21} \cdot \alpha_{1} \cdot \gamma_{1}}{4 \gamma_{1} \gamma_{2}-\gamma_{12} \gamma_{21}}\right)
\end{gathered}
$$


According to Eq. (19) and Eq. (20), the profitability of each player depends on each player's variable cost and price elasticity to demand of the other player. In real world, the relationships are not linear and there are other nonlinear relationships, which could be used such as Multiplicative Competitive Interaction (MCI) and Multinomial Logit (ML) as follows (Elettreby \& Mansour, 2012),

MCI Model : $D_{i}=\frac{M_{i}}{\sum_{j=1}^{n} M_{j}} \times D_{0}=\frac{D_{0} e^{\rho_{i}} \cdot \prod_{k=1}^{K}\left(X_{k i}\right)^{v_{k}} \cdot \varepsilon_{i}}{\sum_{j=1}^{n} e^{\rho_{j}} \cdot \prod_{k=1}^{K}\left(X_{k j}\right)^{v_{k}} \cdot \varepsilon_{j}}$

MNL Model : $\quad D_{i}=\frac{M_{i}}{\sum_{j=1}^{n} M_{j}} \times D_{0}=\frac{D_{0} e^{\left(\rho_{i}+\sum_{k=1}^{K} v_{k} \cdot X_{k i}+\varepsilon_{i}\right)}}{\sum_{j=1}^{n} e^{\left(\rho_{j}+\sum_{k=1}^{K} v_{k} \cdot X_{k j}+\varepsilon_{j}\right)}}$

Since the relationships are nonlinear, taking the derivatives and solving the nonlinear equations are not easy. Using log-centering, one may linearizes the first equation in Eq. (23) as follows (Harsanyi, 2004),

$$
\begin{aligned}
& D_{i}=\frac{M_{i}}{\sum_{j=1}^{n} M_{j}} \times D_{0}=\frac{D_{0} \cdot e^{\rho_{i}} \cdot \prod_{k=1}^{K}\left(X_{k i}\right)^{v_{k}} \cdot \varepsilon_{i}}{\sum_{j=1}^{n} e^{\rho_{j}} \cdot \prod_{k=1}^{K}\left(X_{k j}\right)^{v_{k}} \cdot \varepsilon_{j}} \\
& \log \left(D_{i}\right)=\log \left(R_{i} \times D_{0}\right)=\log \left(D_{0}\right)+\log \left(R_{i}\right) \\
& \Rightarrow \log \left(D_{i}\right)=\log \left(D_{0}\right)+\log \left(\frac{M_{i}}{\sum_{j=1}^{n} M_{j}}\right)=\log \left(D_{0}\right)+\log \left(M_{i}\right)-\log \left(\sum_{j=1}^{n} M_{j}\right) \\
& \Rightarrow \log \left(D_{i}\right)=\log \left(D_{0}\right)+\rho_{i}+\sum_{k=1}^{K} v_{k} \cdot \log \left(X_{k i}\right)+\log \left(\varepsilon_{i}\right)-\log \left(\sum_{j=1}^{n} e^{\rho_{j}} \cdot \prod_{k=1}^{K} X_{k j}^{v_{k}} \cdot \varepsilon_{j}\right)
\end{aligned}
$$

Using a straightforward math yields,

$$
\log \left(\tilde{D}_{i}\right)=\log \left(D_{0}\right)+\bar{\rho}+\sum_{k=1}^{K} v_{k} \cdot \log \left(\tilde{X}_{k}\right)+\log (\tilde{\varepsilon})-\log \left(\sum_{j=1}^{n} e^{\rho_{j}} \cdot \prod_{k=1}^{K} X_{k j}^{v_{k}} \cdot \varepsilon_{j}\right)
$$

where $\tilde{D}_{i}, \tilde{X}_{k}$ and $\tilde{\varepsilon}$ represent the arithmetic mean and $\bar{\rho}$ represents the geometric mean. Differentiating Eq. (26) from Eq. (25) yields,

$$
\log \left(\frac{D_{i}}{\tilde{D}_{i}}\right)=\rho_{i}^{*}+\sum_{k=1}^{K} v_{k} \cdot \log \left(\frac{\tilde{X}_{k i}}{\tilde{X}_{k}}\right)+\varepsilon_{i}^{*}
$$

where

$$
\begin{gathered}
\rho_{i}^{*}=\rho_{i}-\bar{\rho} \\
\varepsilon_{i}^{*}=\log \left(\frac{\varepsilon_{i}}{\tilde{\varepsilon}}\right)
\end{gathered} .
$$

Similarly, we may simplify the MNL model as follows, 


$$
\log \left(\frac{D_{i}}{\tilde{D}_{i}}\right)=\rho_{i}^{*}+\sum_{k=1}^{K} v_{k} \cdot\left(X_{k i}-\bar{X}_{k}\right)+\varepsilon_{i}^{*}
$$

We now demonstrate the proposed study of the paper using a graphical system dynamics representation in Fig. 3 as follows (Kim \& Kim, 1997),

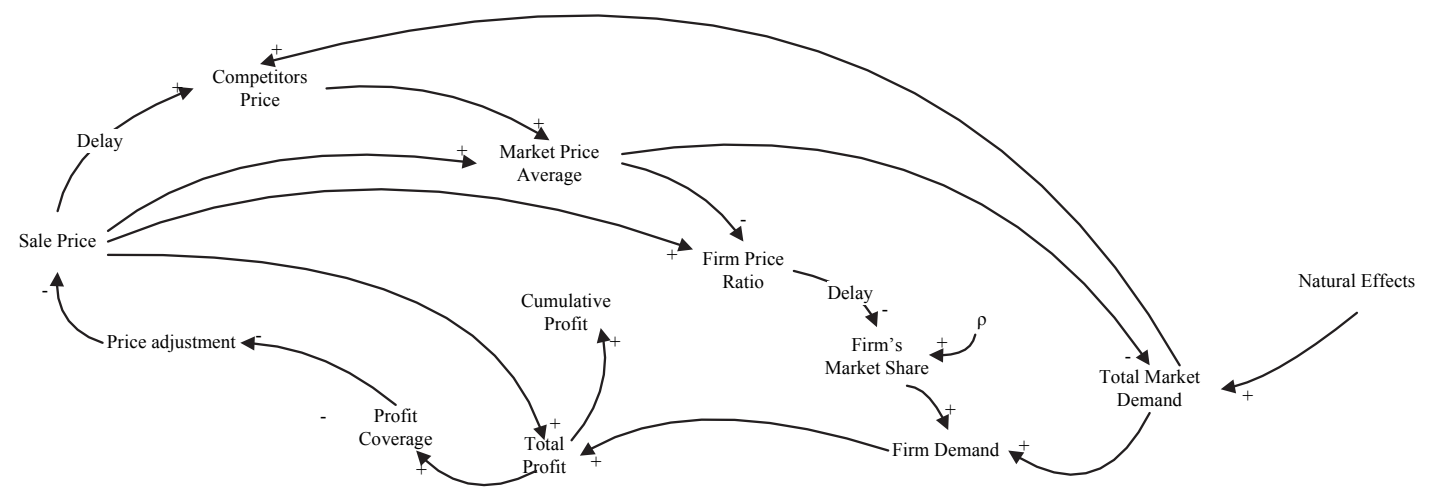

Fig. 3. The cause and effects of price on market share in an oligopoly market

According to Fig. 3, price of each firm is compared with the average price of the market and ultimately determines the market share of each firm. In addition, the role of natural factors in total demand of the market, as well as the impact of the demand on firm's profits and earnings' impact on prices, which is the status of the market is considered. The cause and effect relationships consider the effects of player's pricing strategy on market equilibrium. Note that in this model, we assume all players are looking for fair return considered for each sector of industry. When a firm considers a discount, the firm will expect gaining higher market share and competitors may not react on such discount decisions. In addition, Fig. 4 shows the cause and effects of demand of each firm on profit (loss) in an oligopoly market.

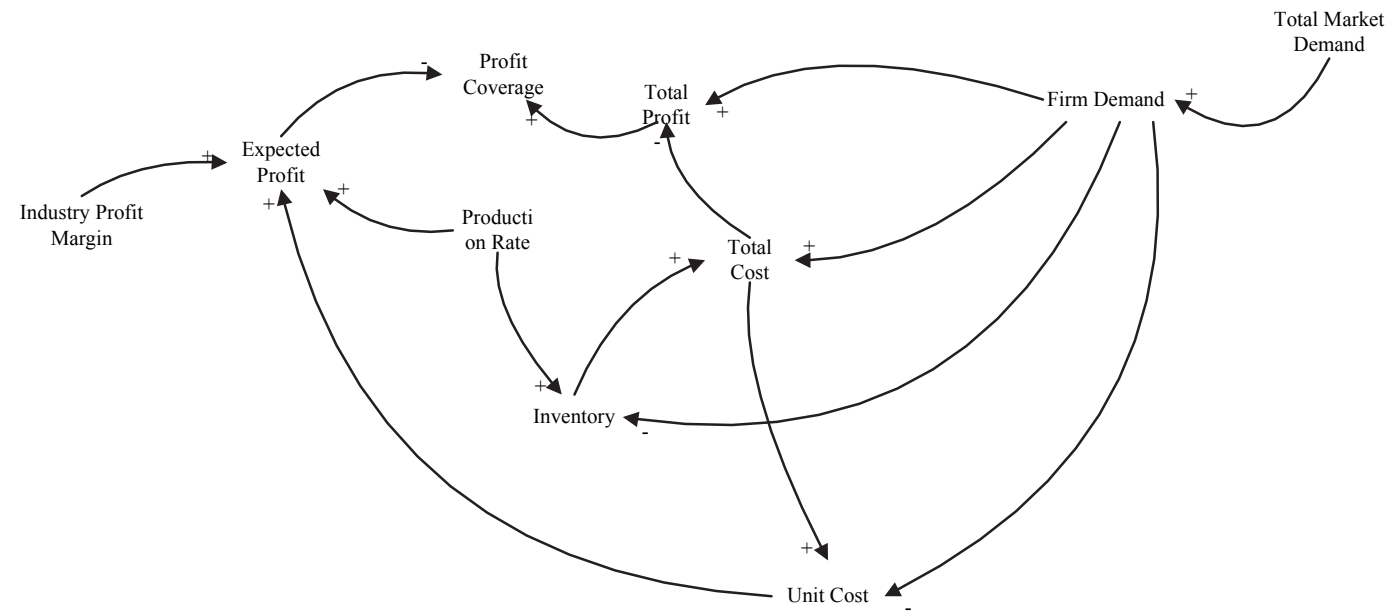

Fig. 4. The cause and effects of demand of each firm on profit (loss) in an oligopoly market

According to Fig. 4, total market demand influences positively on firm's demand and it influences on total profit, total cost and unit cost, accordingly. In addition, an increase on firm demand reduces inventory, which influences positively on total cost. Moreover, an increase on production rate influences positively on inventory and expected profit. Based on the description given in Fig. 3 and Fig. 4 and details of the information provided we present the proposed model in Fig. 5 as follows, 


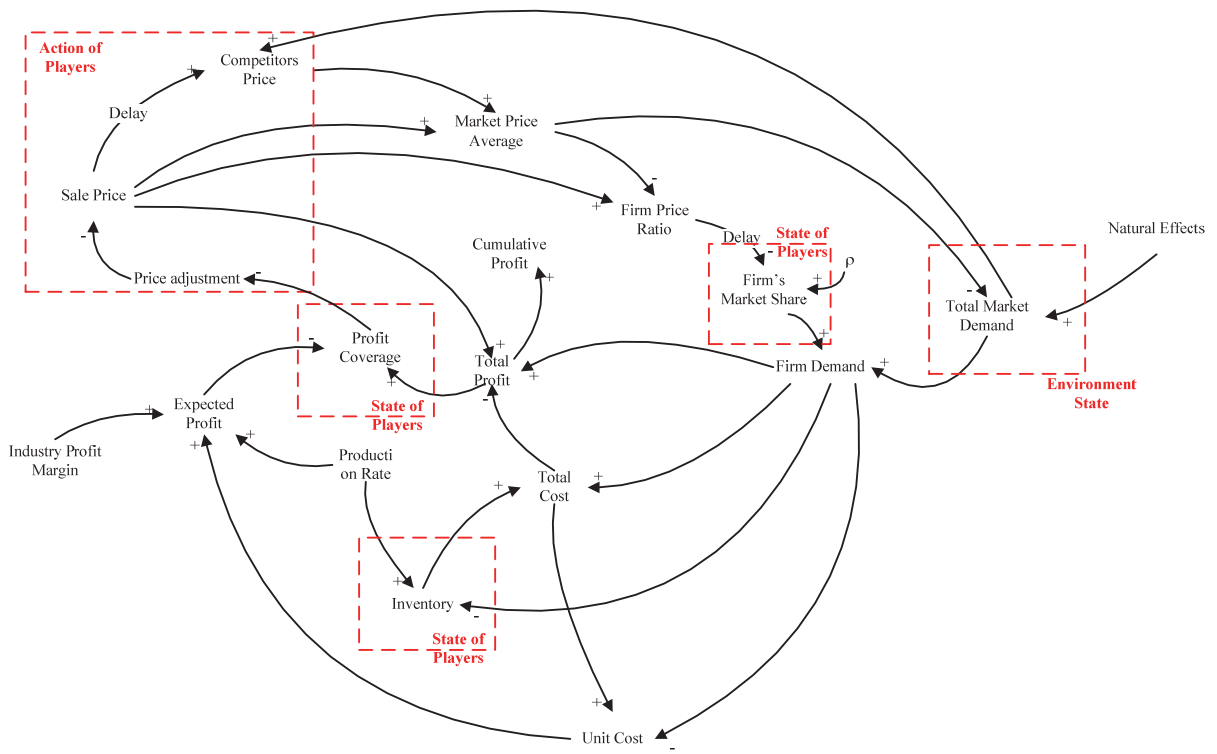

Fig. 5. The proposed cause and effect relationships in oligopoly market

\section{The results}

For the proposed study of this paper, we have considered a market with the following dataset,

Price coefficient $\gamma=0.975$, the effect of firm compared with mean market is 0.65 , which means the proposed firm has better performance compared with other firms on the market, the market is considered on weekly basis and the model has been simulated for 104 weeks or two years. In addition, variable and fixed costs are equal to 100 and 500,000, respectively. Moreover, weekly holding cost is 20 and industry average profit is also $20 \%$. We assume it will take two weeks until other players recognize a player's changing in strategy. There are 8 players on the market and each player produces 41,000 units. Finally, the initial price at the beginning of the planning is set to 200. The simulation is executed in Vensim software and Fig. 6 shows the results of inventory circulation.

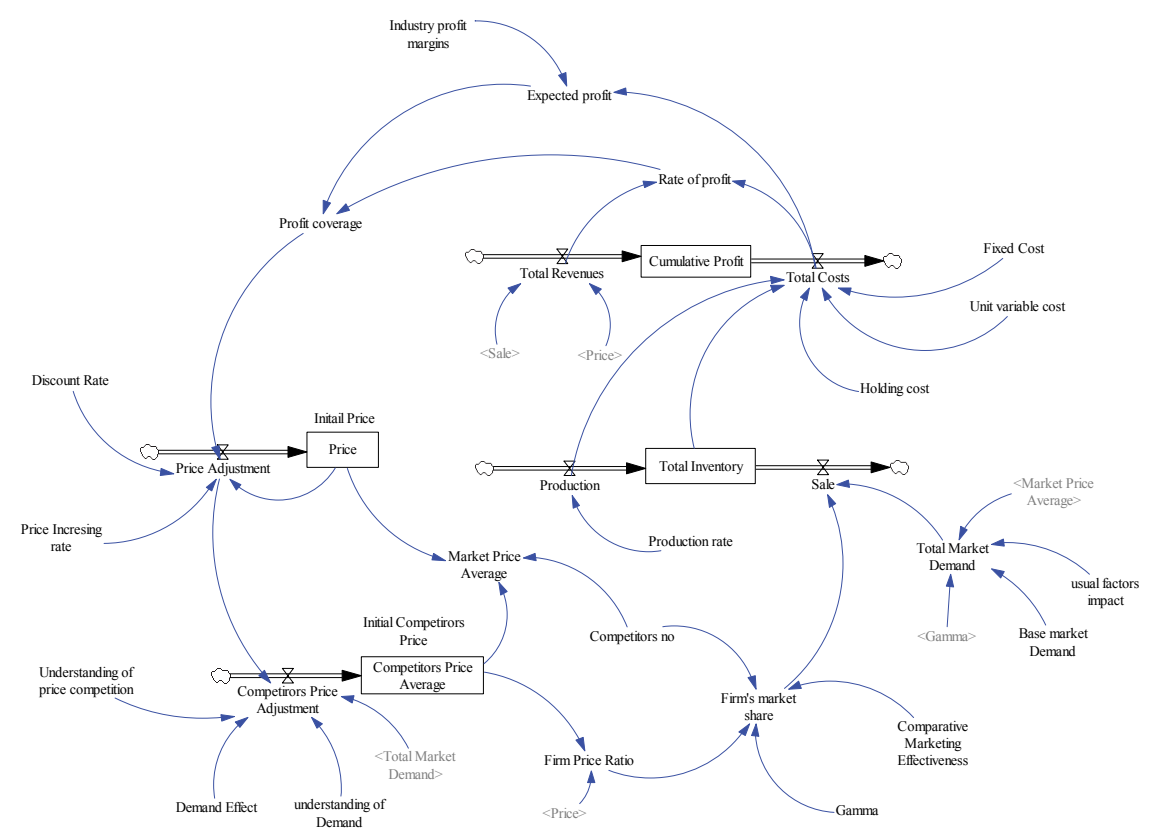

Fig. 6. The output of inventory flow 
As explained earlier, we have been looking on the effect of change on price on other players' behaviors, the effects of price change on other players' decisions. Our results have indicated that all changes will be stabilized over a long period of time. Fig. 7 shows the changes on price of the firm, the change on volume of production over time.

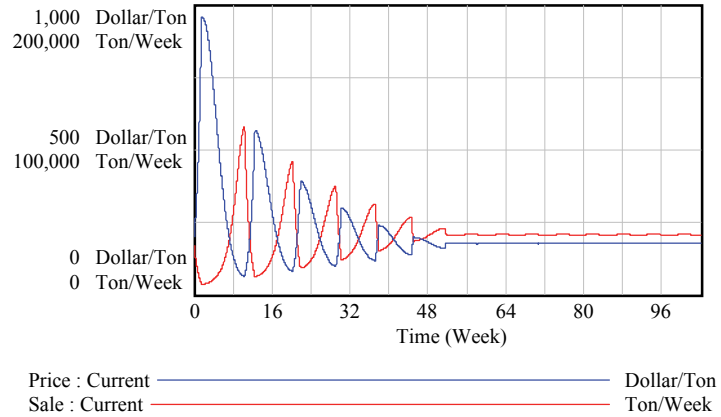

Price versus sales volume over time

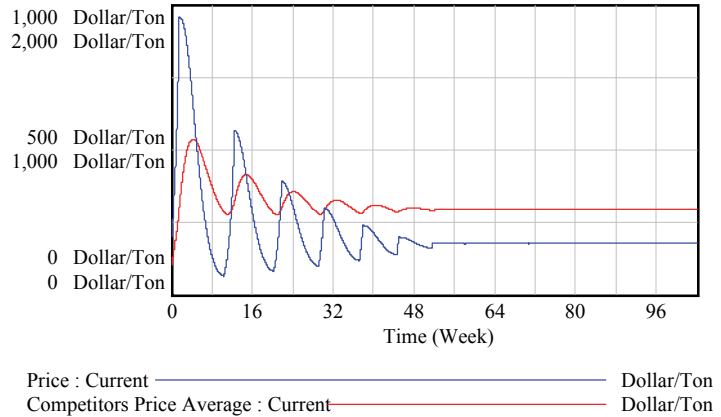

Price versus competitors' prices

Fig. 7. The results of the change on price on other players' production and price

As we can observe from the results of Fig. 7, after approximately one year, the system becomes stable. Fig. 8 also presents the trend on price and sales figures. Once more time, price and sales become stable after one year.

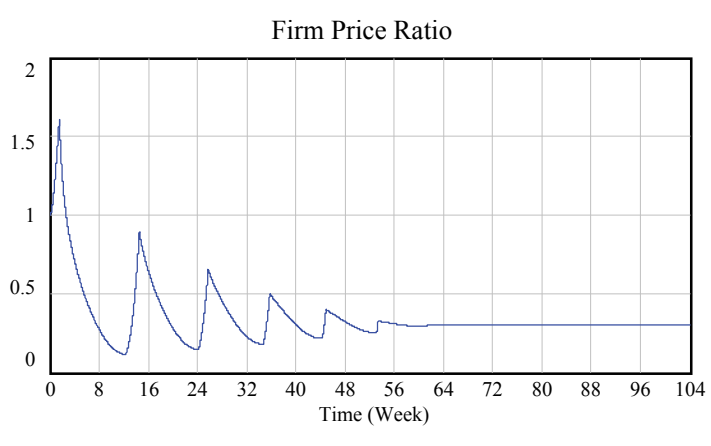

Firm Price Ratio : Current

Firm price ratio

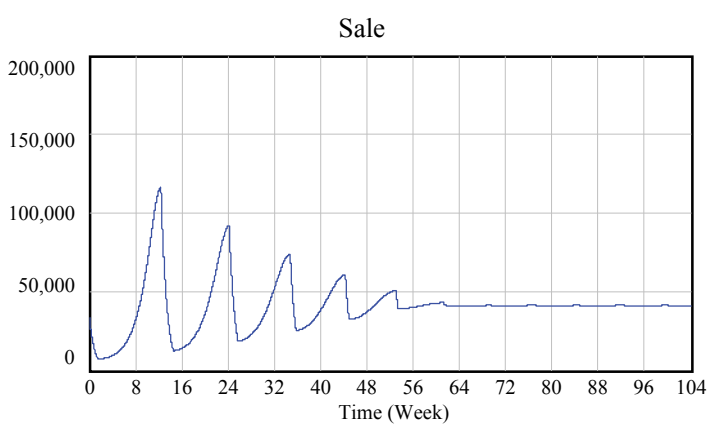

Sale : Current

Sales

Fig. 8. The trend on price and sales figures

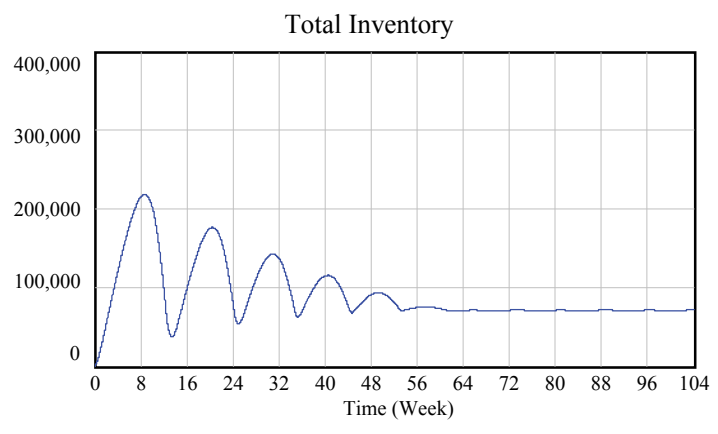

Total Inventory : Current

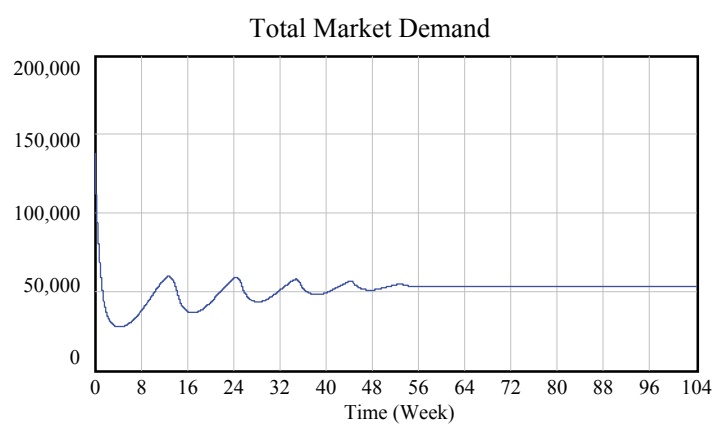

Total Market Demand : Current

Trend of total market demand

Trend of inventory

Fig. 9. The trend on inventory and market demand 
The results of Fig. 9 also shows that inventory and market demand become stable after one year. Finally, Fig. 10 shows the changes on profit and as we can see, although there some fluctuations on profitability but after almost one year, there is steady trend on profitability.

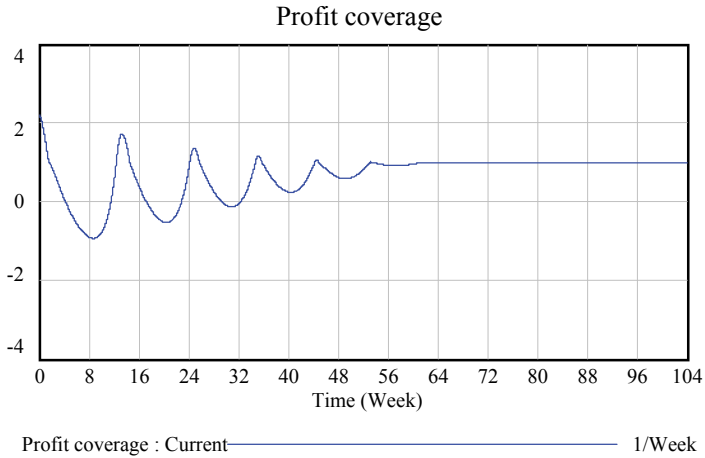

The rate of profit coverage

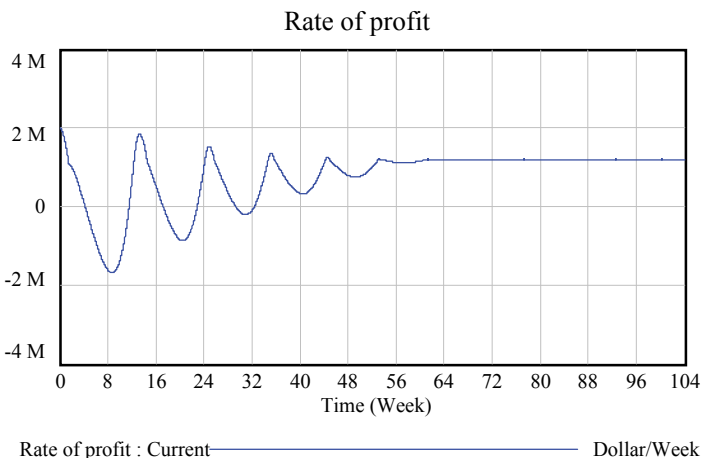

The rate of profit

Fig. 10. The trend on profit coverage and profit

\section{Conclusion}

In this paper, we have presented a hybrid of game theory and dynamic systems to study the behavior of firms in an oligopoly market. The aim of the study was to model a complex strategy for oligopoly game on the basis of feedback loops and system dynamics, explored the dynamics prevailing in a game in the real world. The resulted model has been solved under some special conditions where traditional game theory methods were unable to handle. The method incorporated a combination of qualitative methods including interviews with industry experts to prepare the model and quantitative methods of system dynamics, simulation methodologies and game theory. The results have indicated that competitive behavior and the important parameters such as volume of demand, interest rates and price fluctuation could be stabilized after a transition period.

\section{References}

Ahmed, E., \& Hegazi, A. S. (2006). On dynamical multi-team and signaling games. Applied Mathematics and Computation, 172(1), 524-530.

Akiyama, E., \& Kaneko, K. (2000). Dynamical systems game theory and dynamics of games. Physica D: Nonlinear Phenomena, 147(3), 221-258.

Akiyama, E., \& Kaneko, K. (2002). Dynamical systems game theory II: A new approach to the problem of the social dilemma. Physica D: Nonlinear Phenomena, 167(1), 36-71.

Asker, S. S. (2007). On dynamical multi-team Cournot game in exploitation of a renewable resource. Chaos, Solitons \& Fractals, 32(1), 264-268.

Baiardi, L. C., Lamantia, F., \& Radi, D. (2015). Evolutionary competition between boundedly rational behavioral rules in oligopoly games. Chaos, Solitons \& Fractals, 79, 204-225.

Castanon, D., \& Athans, M. (1976). On stochastic dynamic Stackelberg strategies. Automatica, 12(2), 177-183.

Elettreby, M. F., \& Mansour, M. (2012). On Cournot dynamic multi-team game using incomplete information dynamical system. Applied Mathematics and Computation, 218(21), 10691-10696.

Harsanyi, J. C. (2004). Games with incomplete information played by "Bayesian" players, i-iii: part i. the basic model\&. Management science,50(12 supplement), 1804-1817.

Kim, D. H., \& Kim, D. H. (1997). System dynamics model for a mixed strategy game between police and driver. System Dynamics Review, 13(1), 33-52. 
Lambertini, L., \& Mantovani, A. (2014). Feedback equilibria in a dynamic renewable resource oligopoly: pre-emption, voracity and exhaustion. Journal of Economic Dynamics and Control, 47, 115-122.

Merlone, U., \& Szidarovszky, F. (2015). Dynamic oligopolies with contingent workforce and investment costs. Mathematics and Computers in Simulation,108, 144-154.

Mingers, J. (2004). Realizing information systems: critical realism as an underpinning philosophy for information systems. Information and organization, 14(2), 87-103.

Nash, J. F. (1950). Equilibrium points in n-person games. Proc. Nat. Acad. Sci. USA, 36(1), 48-49.

Nash, J. (1951). Non-cooperative games. Annals of mathematics, 51, 286-295.

West, R. L., \& Lebiere, C. (2001). Simple games as dynamic, coupled systems: Randomness and other emergent properties. Cognitive Systems Research, 1(4), 221-239.

Zhang, N., Yan, Y., \& Su, W. (2015). A game-theoretic economic operation of residential distribution system with high participation of distributed electricity prosumers. Applied Energy, 154, 471-479.

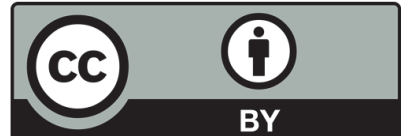

(C) 2016 by the authors; licensee Growing Science, Canada. This article is an open access article distributed under the terms and conditions of the Creative Commons Attribution (CC-BY) license (http://creativecommons.org/licenses/by/4.0/). 